\section{A study to examine the uses of personal strength in relation to mental health recovery in adults with serious mental illnesses: a research protocol}

\author{
Huiting Xie, Peng Yuan, Song Song Cui, \\ Melissa Sng Siok Yen \\ Institute of Mental Health, Singapore
}

\begin{abstract}
This study will explore the relationships among strengths self-efficacy, resourcefulness, stigma experience and mental health recovery in community-dwelling adults with serious mental illnesses. Mental health practices have focued on psychopathphysiology. Stigma heavily plagued clients with mental illnesses and is one of the greatest barriers to mental health recovery. Personal strengths like strengths self-efficacy, people's confidence in using their personal strengths, and resourcefulness, the ability to carry out daily activities, have been linked to positive mental health. However, the linkage between strengths self-efficacy, resourcefulness and mental health recovery remains uncharted. A cross-sectional, descriptive, mixed methods study will be conducted. A funded study by the Sigma Theta Tau, Upsilon Eta Chapter, August 2013 , involving a convenience sample of 100 participants is planned. Included are community dwelling adults between 21 to 65 years old having been diagnosed with serious mental illnesses. Clients with current co-occurring substance abuse will be excluded. Participants complete questionnaires and undergo an interview. Correlations among the study variables will be examined. Regression analysis will determine if recovery can be predicted by strengths self-efficacy, resourcefulness and stigma experience. Interview data will be transcribed and analyzed by thematic analysis. This study will look beyond clients' disability to focus on their recovery and healing capacities such as strengths self-efficacy and resourcefulness. Findings will expand our knowledge about mental health recovery. Knowledge gained from this study may pave the way for future nursing strategies to aid recovery and inform the development of positive, strengths-based interventions.
\end{abstract}

\section{Key points}

\section{Why is this research or review needed?}

Mental health practices are based heavily on pathological thinking, focusing on problems and failures in people with mental illnesses.

Stigma heavily plagued clients and is one of the greatest barriers to mental health recovery.

Personal strengths such as strengths selfefficacy and resourcefulness in people with mental illnesses are seldom explored.

\section{What are the main findings?}

Key findings of this study will go beyond the symptoms and disabilities of people with mental illnesses to focus on their recovery and healing capacities.

We hypothesized that strengths self-efficacy, resourcefulness, stigma experience will be correlated with or predictive of mental health recovery in community dwelling adults with serious mental illnesses.

\section{How should the findings be used to influence policy/practice/ research/education?}

Findings will expand our knowledge about mental health recovery and positive concepts such as strengths self-efficacy and resourcefulness.

Approaching mental illnesses positively may help to reduce stigma that impedes recovery.

Knowledge gained from this study may pave the way for future nursing strategies to aid recovery and inform the development of positive, strengths-based interventions.

\section{Introduction}

Mental illnesses are a cause of concern. Internationally, across countries in America, Europe, Middle East, Africa, and Asia, nine to $17 \%$ of the population surveyed had some episodes of mental illnesses in the previous year. ${ }^{1}$ In the USA, about 57.7 million people, $26 \%$ of adults aged 18 years and older had at least one mental illness in any given year.

Mental illnesses contribute to health burden and disability around the world. 1 The World Health Organization reported that an estimated 873,000 people committed suicide yearly, and more than $90 \%$ of all cases of suicide were associated with mental disorders. ${ }^{2}$ The situation can worsen with the global burden resulting from mental illnesses expected to rise from $12.3 \%$ in the year 2000 to $14.7 \%$ in the year 2020.3

Serious mental illnesses (SMI), like schizophrenia or major depression, significantly increase the risk of early mortality. Every year, more people in Singapore die from suicide than from traffic accidents. 4 In Singapore, about one in 16 people have experienced major depressive disorder some point in their lives while alcohol abuse and obsessive compulsive disorders affect one in 29 and one in 33 people
Correspondence: Huiting Xie, Institute of Mental Health, Singapore, Buangkok Green Medical Park, 10 Buangkok View, 539747 Singapore.

Tel.: +65.6389.2207.

E-mail: hui_ting_xie@imh.com.sg

Key words: Community psychiatric nursing; selfefficacy; service user perspective; adult psychiatric nursing; patient perspectives.

Acknowledgements: the study team would like to acknowledge the Institute of Mental Health, Singapore, Singapore Anglican Community Services and Dr Jaclene Zauszniewski, Case Western Reserve University, America, for rendering their support to this proposed study.

Contributions: the authors contributed equally.

Conflicts of interest: the authors declare no potential conflict of interest.

Funding: this study is supported by the Sigma Theta Tau, Upsilon Eta Chapter Research Grant (August 2013).

Received for publication: 31 December 2013.

Revision received: 17 February 2014.

Accepted for publication: 17 February 2014.

This work is licensed under a Creative Commons Attribution NonCommercial 3.0 License (CC BYNC 3.0).

(C) Copyright H. Xie et al., 2015

Licensee PAGEPress, Italy

Health Psychology Research 2015; 3:2209

doi:10.4081/hpr.2015.2209

respectively. The situation is worsen as stigma associated with mental illnesses inhibits the public in Singapore less willing to pay for mental healthcare services, and often leads to the segregation of people with mental health conditions away from the public. ${ }^{4}$

\section{Background}

Influenced by the mass appeal of the Diagnostic and Statistical Manual of Mental Disorders (DSM), mental health practices are heavily aligned with pathological thinking, focusing on human problems and failures. ${ }^{5}$ In the past, serious mental illnesses were viewed pessimistically, with the course of illness being deteriorative and treatment being stabilization of the illness condition at best even if outcomes were not all bad. 6,7

Little attention has been given to positive approaches to enhance mental health. ${ }^{8}$ As health providers paint a gloomy picture of SMI as chronic and irreversible, people with SMI 
also view themselves negatively. Many of them internalize the stigma and perceive themselves negatively,9,10 leading to decreased psychological health (e.g. lowered self-esteem) and impaired role performance.

Recovery from mental illnesses has to be the guiding vision for mental health services. ${ }^{6}$ The National Institute of Mental Health states promotion of recovery, not merely the reduction of symptoms, as their ultimate goal.11 For people with mental illnesses, recovery gives them a sense of hope that they can continue to lead normal lives despite their illnesses. ${ }^{12}$ However, psychopathology is still very much evident in mental health practices today and we are still a distance away from achieving the vision of recovery. ${ }^{7}$

Stigma is one of the biggest barriers to recovery. America's Surgeon General stated that stigma should not be tolerated to reduce the illness burden and improve recovery. ${ }^{11}$ The mental health blueprint in Singapore highlights the need to build resilience and reduce stigma to achieve recovery. ${ }^{4}$

While stigma impedes recovery, personal strengths have been linked to mental health. ${ }^{13,14}$ Personal strengths are the person's positive traits that either create a sense of personal accomplishment or enhance their abilities to provide near perfect performance in a specific task. ${ }^{14,15} \mathrm{~A}$ large-scale web-based study found that recovery from psychological disorders was associated with greater personal character strengths. ${ }^{13}$ Additionally, results from another study suggested that clients engaged in a recovery orientated program that emphasized the development and use of personal strengths had better recovery. ${ }^{16}$

Resourcefulness, the ability to carry out daily activities by themselves (personal resourcefulness) or by seeking help (social resourcefulness), has also been linked to improvements in mental health. In a study of 104 cognitively intact elders dealing with the stressor of relocation, resourcefulness made the relocation process more psychologically pleasant. ${ }^{17}$ In two studies relating resourcefulness to depression in the caregivers' population, resourcefulness was negatively related to depressive symptoms. 18,19

\section{Research gaps}

Even if personal strengths have been linked to mental health, 13,14 the impact of these in people with SMI is not known as mental health practices still tends to be focused on investigating problems in the person, rather than their personal strengths. Little is known about the perceived ability to use personal strengths (strengths self-efficacy) in people with mental health issues. Resourcefulness has also neither been examined in people with mental health issues nor in relation to mental health recovery.

\section{Theoretical framework}

The Community Vulnerability (CV) framework guides this study. The CV model has three main constructs: stressors, assets and outcomes. Stressors and assets both impact the outcome. When assets are low and stressors are high, the community becomes vulnerable to adverse outcomes..$^{20}$ If assets are strong enough to overcome stressors, then the outcome can be favorable. In this study, the CV model is applied to individuals in the community with mental health recovery as the outcome. Stigma is conceptualized as a stressor that hinders recovery while personal strengths like strengths self-efficacy and resourcefulness are conceptualized as human assets that when sufficient are able to overcome the effects of stressors, leading to desirable outcome like recovery.

\section{Significance}

This study goes beyond the symptoms and disabilities of people with SMI to focus on their recovery and healing capacities. The focus is on positive attributes such as strengths selfefficacy, resourcefulness and whether they influence mental health recovery.

Personal strengths are newer concepts in the psychiatric arena and the findings may be a step towards expanding the knowledge base for positive concepts such as strengths selfefficacy, resourcefulness, and mental health recovery. Approaching mental illnesses positively may also help to reduce the stigma that impedes mental health recovery. Knowledge gained from this study may pave the way for future nursing strategies to aid mental health recovery.

\section{The study}

\section{Aims}

This study aims to examine if the use of personal strengths influence mental health recovery in adults with serious mental illnesses. The major variables under study are: i) strengths self-efficacy, ii) resourcefulness, iii) stigma experience and iv) mental health recovery.

The following research questions will be addressed. In community-dwelling adults with serious mental illnesses (SMI); i) What are the associations among strengths self-efficacy, resourcefulness, stigma experience and mental health recovery? ii) Do strengths self-efficacy, resourcefulness, and stigma experience predict mental health recovery? iii) If predictions are significant, do the predictions still hold after controlling for related socio-demographic covariates? iv) How do participants use their personal strengths pertaining to their mental health?

\section{Design}

A cross-sectional, descriptive, predictive, mixed methods design will be used in this study that is set in the Singapore community. Literature on the variable strengths self-efficacy is limited, and mental health recovery and stigma experience has not been examined much from the patients' perspective. Since little is known about the phenomenon, a descriptive study design allows a large amount of information to be collected quickly and economically. ${ }^{21}$ However, one setback of a descriptive design using surveys, is that information collected can be superficial. ${ }^{21}$ Hence, this study will include a qualitative component as part of a mixed method study design so that words or narrative information generated from the study can complement the quantitative analysis of surveys. ${ }^{22} \mathrm{~A}$ cross-sectional study design measures all the variables at a single time point. It is well-known that people with mental illnesses often have trouble adhering to treatment and follow-up regime. The attrition rates of participation may be high and the power of the study diminishes with decreasing sample size if this was to be a longitudinal study. In addition, the main outcome of interest, recovery, in the study though may fluctuate but does not change greatly over a short period of time, hence a cross-sectional study design will be able to capture the phenomenon.

This study will also involve a mixed-methods approach. The quantitative aspect of the study will explore relationships among strengths self-efficacy, resourcefulness, stigma experience, and mental health recovery. The prediction of mental health recovery from the variables strengths self-efficacy, resourcefulness and stigma experience will also be examined. Secondary research questions will be answered using qualitative descriptive methodology in which participants will be asked opened-ended questions regarding the use of their personal strengths. The qualitative data collected will be used to complement the quantative data collected to describe participants' personal strengths and the use of their personal strengths pertaining to their mental health.

\section{Participants}

A convenience sample will be obtained. Participants included are community-dwelling adults having been diagnosed with SMI. Selecting the sample from the community keeps the bias of acute psychotic symptoms from affecting participants' perception of their recovery.

Power analysis was conducted $a$ priori based on a medium effect size as attained from previous studies, ${ }^{13,23}$ using $\mathrm{G}^{*}$ Power. A total of 67 participants is required, assuming an alpha level of $\mathrm{P}=0.05$ and a power of 0.80 . To be conservative with the aim of recruiting a larger 
sample and to account for attrition, we aim to recruit 100 participants.

\section{Inclusion criteria}

To be included in this study, participants should be community-dwelling individuals. People in the community have fewer unmet needs in the areas of psychological distress and psychotic symptoms than those in hospital-based facilities. ${ }^{24}$ Participants included in this study are adults 21 to 65 years old (both inclusive) having been diagnosed with having SMI. Participants should be mentally stable enough to understand the nature and purpose of the study. They should also be able to read the questionnaire or understand what is spoken to them from the questionnaire. Their capacity to understand will be assessed by giving them information regarding the study to read or after listening to the researcher read the information, followed by them giving an account of what the gist of the study is. The information are based on the patient information sheet written in English and can be explained verbally in other languages.

\section{Exclusion criteria}

People who are currently abusing substances are excluded from the study. It has been well established that the prevalence of psychoactive substance abuse is high among people with mental illnesses like schizophrenia. 25 Research indicates that people with cooccurring substance abuse are highly prone to relapse to substance abuse, even after they have attained full remission of their mental illnesses. They may also lack independent housing, meaningful activities and social supports which could be necessarily for recovery. 26 Hence, the recovery of people with SMI and cooccurring substance abuse may be affected. Since this study examines recovery after mental illness, people with current substance abuse are excluded from the study.

\section{Instruments}

Strengths self-efficacy will be measured by the Strengths Self-efficacy Scale (SSES) that was developed by Chaichanasakul et al. ${ }^{27}$ Content validity of SSES was assessed independently by four experts in positive psychology. SSES was tested out with 214 participants in the community, and yielded a high level of internal consistency (reliability) Cronbach's alpha is 0.97 .27

Resourcefulness will be measured by the Resourcefulness Scale (RS) developed by Zauszniewski et al.28 RS's items had been examined in conjunction with the Self-control Schedule and the Help Seeking Resource Scale, well-known measures of personal and social resourcefulness respectively giving RS its construct validity. RS was tested on 451 chronically ill elders yielding a high level of internal consistency (cronbach's alpha=0.85).
Perception of personal mental health recovery will be measured by the Mental Health Recovery Measure (MHRM) developed by Young et al. ${ }^{29}$ MHRM had content validity clients since it was developed using narrative data provided by clients with psychiatric disabilities. Convergent validity of MHRM has been demonstrated with instruments assessing empowerment, resilience and community living ability. ${ }^{30}$ MHRM is suitable for use with the ethnically diverse population in Singapore, as it has been tested with clients from several ethnic groups with no significant differences in mean MHRM scores between different ethnic groups. Good internal consistency has been demonstrated with 279 clients (cronbach's alpha=0.93). ${ }^{30}$

Stigma experience will be measured by the Stigma Scale (SS) developed by King et al.31 The SS assesses stigma experienced by the person with mental illness themselves rather than from a third person's viewpoint. The SS is a valid measure for the person with mental illness as it was developed after in-depth interview with 46 clients in community mental health services. ${ }^{32}$ Discriminant validity was shown when SS was negatively correlated with global self-esteem. As in this study, where participants are recruited from the community with different SMI diagnoses, the SS had also been tested on 193 participants with varied settings and diagnoses. Good internal consistency has been demonstrated with 193 clients (cronbach's alpha=0.87). ${ }^{31}$

An interview guide was developed with open-ended questions for the qualitative component for this research study. A prior review of the literature was done to identify the phenomenon that needed further exploration using qualitative methodology. Questions were developed in the interview guide to examine the phenomenon of mental health recovery from participants' point of view. The literature offered supporting evidence regarding the contributions of personal strengths towards positive outcomes, but little is known about personal strengths in people with mental illnesses. Even more scarce was evidence linking personal strengths directly to the recovery of people with mental illnesses. Hence, the interview guide also included questions exploring situations that participants use their personal strengths and the relationships between these strengths and their mental health recovery. These questions were developed by the principal investigator for this research study and reviewed by an experienced qualitative researcher, and a faculty member at a renowed university to ensure its validity to the purpose of this study.

\section{Data collection}

After obtaining ethical clearance to commence the study, a pilot study will be conduct- ed on about 5 to 10 people with serious mental illness who meet the inclusion and exclusion criteria to test the feasibility of using the instruments in this study and the feasibility of conducting the full-scale study. They will fill up the instruments and provide their opinions about the instruments. Following which, the researchers will get together as a team to review the feedback of responents to the pilot study. The research team will decide if changes to the instruments were needed. If changes to the instruments were needed, the modified instruments will be sent back to the original authors of the instruments for their review and the ethics review board will be informed of the amendments to the study. Study amendments will also be done if the instruments were found not to be feasible for this study and new instruments were to be used. If no changes were made to the instruments and the researchers find that it is feasible to continue, the full scale study will be conducted.

With permission, posters will also be put up and flyers distributed at community mental health agencies like day care centers, or clubhouses, outpatient clinics around Singapore. These agencies only accepts patients who have been diagnosed with serious mental illnesses. Researchers just provide their contact information without seeking the records of potential participants. Potential participants who were interested in the study contact researchers on their own accord. This minimizes coercion of participating in the study.

Potential participants will approach the researchers directly or express their interest to participate through telephonic or electronic communication after reviewing the posters and flyers. The principal investigator will then screen the potential participants for their eligibility to participate in the study based on the inclusion and exclusion criteria. Only the principal investigator will be involved in the screening to minimize bias. If the potential participants were eligible and were interested to participate, they will meet with a researcher at a mutually agreed upon time and venue for informed consent taking and data collection.

The researcher will be trained prior to commencement of the study obtain consent, administer the instruments and conduct interviews with participants. The instruments will also be administered to all participants in a specified order to control for testing effects. The Mental Health Recovery Measure which assesses recovery, the main outcome of interest will be administered first. This is followed by the Strengths Self-efficacy Scale. Participants will be shown the definition of personal strengths and then asked to list their strengths. If they have difficulty identifying their strengths, they will be asked to think about situations when they have felt having 
accomplished something. Then, participants will answer questions regarding the use of their personal strengths in different situations. Subsequently, the Resourcefulness Scale and the Stigma Scale will be administered to the participants, followed by the questionnaire to assess their socio-demographic information. All the instruments will be administered in the form of a structured interview where the researcher or data collectors will read out the questions and record participants' response. Administering the questionnaires in the form of a structured interview greatly increased the response rate about $80 \%$ as compared to mailed or web-based questionnaires that typically obtained responses rates of $50 \%$ or lower. ${ }^{33}$ In addition, the structured interview also gives researchers control over the orders which questions are answered and minimize the possibility of having missing responses, thus reducing bias in the results. ${ }^{33}$

Should the participant ask for clarification, the researchers or data collectors will only repeat the questions or define the terminology in a pre-determined manner but reframe from giving further interpretation. This is to ensure that the same information will be transmitted to the participants in a similar manner whether it is the principal investigator, researchers or data collectors who will be meeting with the participants. It is also for this reason that inter-rater reliability will not be assessed as the questions are already stated in the instruments and the researchers or data collectors are merely present to deliver the instruments to participants.

A short interview will follow the questionnaires administration. The principal investigator or her representative will ask participants' questions according to the interview guide. Participants are again asked to base their answers on their own views and to answer truthfully without any concern for incorrect answers as there are no right or wrong answers. Having such reassurance may increase the chance that participants will respond truthfully, thus enhancing the chance that data valid to the participants are collected. To further increase the validity of the data, researchers will verify his/her understanding of participants' response verbally before the responses are recorded, thus giving the participants an opportunity to correct any errors before records are finalized. This provides an opportunity for participants to recognize the responses to be true to them, hence increasing the credibility of the findings later on. ${ }^{34}$

\section{Data analysis}

Social Package for Statistical Science will be used for data analysis. Descriptive statistics will describe the demographic characteristics of the sample. Reverse scoring will be done as needed and the mean scores of strengths self- efficacy, resourcefulness, mental health recovery, and stigma experience score will be noted. Missing values will be assessed to determine if the missing cases are missing at random where the results of the study will not be biased. Researchers will also ensure that the assumptions of statistical procedures are met. The probability value, $\mathrm{P}$ will be set at 0.05 . Results are considered significant when $P$ is below 0.05 .

Correlation analysis such as Pearson Product Moment Correlation will explore the relationships among i) strengths self-efficacy, ii) resourcefulness, iii) stigma experience and iv) perception of personal mental health recovery and socio-demographic variables.

Linear regression will examine the predictions of i) strengths self-efficacy, ii) resourcefulness, iii) stigma experience on iv) perception of personal mental health recovery. If predictions are significant, socio-demographic covariates that were correlated with the outcome of iv) perception of personal mental health recovery will be entered as a second step to the regression model to examine if the predictions still hold after adjusting for the presence of the socio-demographic covariates.

Qualitative analysis will be done for the secondary research questions of how participants use the personal strengths pertaining to their mental health. The recorded responses will transcribed for analysis. Analysis of the transcript took place over a number of phases as in the process of thematic analysis outlined by Braun and Clarke. ${ }^{35}$ In phase 1, the entire transcript was read through to gain an overview of participants' responses. This was followed by phase 2 where categories or codes were generated for meaning units of data that represented a certain idea. The transcript was rearranged such that extracts of participants' responses representing each category were placed together. The number of extracts and the number of participants who provided the extracts were noted. In the third phase, the categories were reviewed to derive at potential themes. Themes are the broader, over-arching construct that represent units of data. Six potential themes namely, i) use of personal strengths to understand other people, ii) use of compassion to help other people, iii) use of personal strengths to work, iv) use of personal strengths for personal growth, v) use of personal strengths for individual development, vi) use of personal strengths for mental health were derived. The data was collated within the potential themes identified. The transcript was again re-arranged such that extracts representing similar themes were placed together. In phase 4 , these potential themes were further refined. Every category of extracts that fell under each potential theme was reviewed again to determine if the theme was represented in the data. Potential themes with too little data to support the themes were combined and renamed. The principal investigator then re-read the transcript with the themes in mind to ensure accuracy. The process of analysis might also be repeated and themes revised so that the themes could accurately reflect the meaning of the data in the end.

\section{Ethical considerations}

Ethical clearance will be sought $a$ priori. Information about the study will be provided. Participation is entirely voluntary. Refusal or withdrawal from the study will not affect the healthcare services provided. Some people may experience discomfort answering questions for this study. If need be, referral may be made to mental health professionals. Identifying information found in the consent forms will be kept under lock and key. Data collection forms will be de-identified.

\section{Rigor}

This study was designed to enhance validity without compromising the feasibility of conducting the study. Employing a cross-sectional study design with convenience sampling in this study though provide researcher little control over events that occurred, unlike an experimental study, it can overcome several threats to the internal validity of the study to show that the link (relationships or predictions, if established) between the independent variable and dependent variable is true without the interference of other events. According to Polit and Beck, ${ }^{33}$ pre-test post-test study design is most likely to be affected by the threat to history, maturation, testing and instrumentation while experimental study can most likely to be affected by the threat to mortality. Although the cross-sectional design limits the examination of changes over a period of time, such a design prevents many of these other threats to the internal validity of the study.

External validity deals with whether the results of the study can be generalized to other persons, places or times. ${ }^{21}$ However, controlling for one type of validity interferes with the possibility of achieving another type of validity. For example, having a homogenous sample in the study strengthens internal validity but can limit external validity. 33 In this study, convenience sampling will be used without excessive control over the characteristics of participants to be included in the study, so that participants still bear some characteristics of the target population of adults with serious mental illnesses. Furthermore, this study will take place largely in the natural setting without manipu- 
lation of variables as in an experimental study, further enhancing the generalizability of the results.

The rigor of study data could also be determined by its credibility, dependability, confirmability and transferability. 34 A mixed method study design allows for qualitative data to complement the superficial information that are typical in a survey instrument, further enhancing the credibility of the study. Selfreported data collected in this study could be most valid to the participants' experiences and researchers taking the extra step of verifying participants' responses (also known as member checking) enhanced the credibility of the results. ${ }^{33}$

The same research team will analyze the data to enhance internal consistency and helped to ensure that the results of analysis remained stable for every participant, hence enhancing its dependability. With regards to confirmability, the methods for conducting this study will be stipulated in the protocol prior to study commencement and will be closely adhered to. This will leave an audit trail for future researchers to replicate the study to confirm the findings further.

Transferability of the data can be enhanced when researchers give a detailed explanation of the results including the socio demographic characteristics of the participants in this study later on in the research report so that future researchers can get a sense of what kind of context or population the results can apply to. ${ }^{33}$

\section{Discussion}

It is well documented that the experience of stigma has been plaguing people with mental illnesses and impeding their recovery. This study will not only examines stigma and recovery from the perspective of people with mental illnesses as opposed to a third party but also allow the examination of positive concepts such as strengths self-efficacy and resourcefulness capabilities of people with mental illnesses. However, research participants may not benefit directly from participating in this study. Hence, in promoting the study to potential research participants, this would have to be presented to participants. Nevertheless knowledge gained from this study may pave the way for positive strengths-based nursing strategies to be developed to aid mental health recovery, possibly leading to a more comprehensive recovery program for people with mental illnesses. This may in turn benefit participants in future when patients can choose from a greater variety of treatment strategies.

Despite this being a non-invasive study, participants risks associated with daily living may still be expected to occur during the study.
Participants will be advised beforehand to inform the principal investigator or her representative if they feel uncomfortable or become distressed while answering a certain question. If participants were to become distressed in the process of answering questions for the research study, they would be given a break till they are ready to continue or decide to withdraw from the study. Participants may also be given contact information of mental health services where participants can approach for help when they need it.

\section{Limitations}

There are limitations inherent to convenience sampling in the local setting. In particular, with the limited sample size and use of convenience sampling in the local setting, generalizing the findings of the study beyond the context requires further scrunity. As the study will be conducted in the community setting, participants may not be hidden from the public's eye. Participants are asked to choose a private venu where they are comfortable with filling up the questionaires and undergoing the interview.

\section{Conclusions}

The personal strengths of human beings may be illuminated in adversities like mental illnesses. After years of studying human weaknesses and psychological pathology, this study represents a preliminary attempt in the scientific pursuit of human strengths that brought about positive outcome in adversities. Findings could expand our knowledge about recovery from mental illnesses by focusing on personal strengths and positive concepts such as strengths self-efficacy, resourcefulness and mental health recovery.

Findings from this current study could aid in propelling the field of psychiatry away from deficit preoccupation to positive qualities of individuals with mental health issue such as strengths self-efficacy and resourcefulness. Approaching mental illnesses positively could help to reduce the stigma that impeded the recovery of people with mental illnesses.

\section{References}

1. The WHO World Mental Health Survey Consortium. Prevalence, severity, and unmet need for treatment of mental disorders in the World Health Organization world mental health survey. JAMA 2004;291:2581-90.

2. World Health Organization. World Mental Health Day 2006: Building Awareness -
Reducing Risks: Suicide and Mental Illness. Available from: http:/www.who.int/mediacentre/news/rele ases/2006/pr53/en/index.html

3. Murray CJL, Lopez AD, eds. Global burden of disease. A comprehensive assessment of mortality and disability from diseases, injuries, and risk factors in 1990 and projected to 2020. Geneva: WHO; 1996.

4. Chong SA. Mental health in Singapore: a quiet revolution? Ann Acad Med 2007;36: 795-6.

5. Saleebey D. The strengths perspective in social work practice. 4th ed. Boston: MA: Pearson/Allyn \& Bacon; 2006.

6. Anthony WA. Recovery from mental illness: the guiding vision of the mental health service system in the 1990s. Psychosom Rehabil J 1993;16:11-23.

7. Farkas M. The vision of recovery today: what it is and what it means for services. World Psychiatry 2007;6:68-74.

8. Cloninger CR. The science of well-being: an integrated approach to mental health and its disorders. World Psychiatry 2006;5:71-6.

9. Yang LH. Application of mental illness stigma theory to Chinese societies: synthesis and new directions. Singapore Med J 2007;48:977-85

10. Link BG, Struening EL, Neese-Todd S, et al. Stigma as a barrier to recovery: the consequences of stigma for the self-esteem of people with mental illnesses. Psychiatr Serv 2001;52:1621-6.

11. Department of Health and Human Services, DHHS. Mental Health: a report of the surgeon general-executive summary. 1999. Available from: http://mentalhealth. about.com/library/sg/blsummary.htm

12. Oades LG, Deane FP, Crowe TP, et al. Collaborative recovery: an integrative model for working with individuals who experience chronic and recurring mental illness. Australasian Pshychiatry 2005; 13:279-84

13. Peterson C, Park N, Seligman MEP. Greater strengths of character and recovery from illness. J Posit Psychol 2006;1:1726.

14. Epstein MH, Ruldolph S, Epstein AA. Strength based assessment. Teaching Exceptional Children 2000;32:50-4.

15. Hodges TD, Clifton D0. Strengths-based development in practice. In: Linley PA, Joseph S, Eds. Positive psychology in practice. Hoboken, NJ: John Wiley \& Sons Inc; 2004. pp 256-268.

16. Bullock WA, Sage J, Hupp D, et al. Mental health, from illness to wellness: an evaluation of Ohio's wellness management and recovery program in community mental health and consumer-operated service agencies. New Res Ment Health 
2009;18:312-21.

17. Bekhet AK, Zauszniewski JA, Wykle ML. Milieu change and relocation adjustment in elders. West J Nurs Res 2008;30:113-29.

18. Musil C, Warner C, Zauszniewski J, et al. Grandmother caregiving, family stress and strain and depressive symptoms. West $\mathrm{J}$ Nurs Res 2009;31:389-408.

19. Zauszniewski JA, Bekhet A, Suresky M. Relationships among perceived burden, depressive cognitions, resourcefulness, and quality of life in female relatives of seriously mentally ill adults. Issues Ment Health Nurs 2009;30:142-50.

20. Alasia A, Bollman R, Parkins J, Reimer B. An index of community vulnerability: Conceptual framework and application to population and employment changes. Research Report N. 088. 2001. Available from: http://dsppsd.pwgsc.gc.ca/collection_2008/statcan/21-601-M/21-601MIE2008088.pdf

21. Schneider Z, Elliott D, Lo-Biondo-Wood G, Haber J. Nursing research: methods, critical appraisal and utilization. New South Wales, Australia: Elsevier; 2003.

22. Johnson RB, Onwuegbuzie AJ. Mixed methods research: a research paradigm whose time has come. Educat Res 2004;33:14-26.
23. Zauszniewski JA, Picot SJ, Roberts BL, et al. Predictors of resourcefulness in African American women. J Aging Health 2005;17:609-33.

24. Killaspy H, Rambarran D, Bledin K. Mental health needs of clients of rehabilitation services: a survey in one trust. J Mental Health 2008;17:207-18.

25. Dervaux A, Laqueille X, Bourdel MC, et al. Cannabis and schizophrenia: demographic and clinical correlates. Encephale 2003;29:11-7.

26. Drake RE, Wallach ME, McGovern MP. Special section on relapse prevention: future directions in preventing relapse to substance abuse among clients with severe mental illnesses. Psychiatr Serv 2005;56:1297-302.

27. Chaichanasakul A, Tsai CL, Zhao R, et al. Development and initial validation of the strengths self-efficacy scale. Doctoral dissertation. University of Missouri and Clifton Strengths School \& Gallup, Columbia, MO. 2009.

28. Zauszniewski JA, Lai CY, Tithiphontumrong S. Development and testing of the resourcefulness scale for older adults. J Nurs Measur 2006;14:57-68.

29. Young SL, Ensing DS. Exploring recovery from the perspective of people with psychi- atric disabilities. Psychiatr Rehabil J 1999;22:219-31.

30. Bullock W.A. Mental health recovery measure. In: Campbell-Orde T, Chamberlin J, Carpenter J, Leff HS., Eds. Measuring the promise of recovery: a compendium of recovery measures. Cambridge, MA: The Evaluation Center; 2005.

31. King M, Dinos S, Shaw J, et al. The stigma scale: development of a standardised measure of the stigma of mental illness. Br J Psychiatry 2007;190:248-54.

32. Dinos S, Stevens S, Serfaty M, et al. Stigma: the feelings and experiences of 46 people with mental illness. Br J Psychiatry 2004;184:176-81.

33. Polit DF, Beck CT. Nursing research: principles and methods. 7th ed. Philadelphia: Lippincott Williams \& Wilkins; 2004.

34. Speziale HJS, Carpenter DR. Qualitative research in nursing: advancing the humanistic imperative. 4th ed. Philadelphia: Lippincott Williams \& Wilkins; 2007.

35. Braun V, Clarke V. Using thematic analysis in psychology. Qual Res Psychol 2006;3:77101. 